Cite this: Nanoscale, 2013, 5, 5905

Received 4th February 2013

Accepted 14th April 2013

DOI: $10.1039 / c 3 n r 00637 a$

www.rsc.org/nanoscale

\title{
Ultrasensitive surface-enhanced Raman scattering detection of trypsin based on anti-aggregation of 4-mercaptopyridine-functionalized silver nanoparticles: an optical sensing platform toward proteases $\uparrow$
}

\author{
Lingxin Chen, ${ }^{\text {*a }}$ Xiuli Fu ${ }^{\mathrm{ab}}$ and Jinhua $\mathrm{Li}^{\mathrm{a}}$ \\ In this work, a simple and sensitive surface-enhanced Raman scattering (SERS) strategy was developed for \\ recognition and detection of trypsin, by using anti-aggregation of 4-mercaptopyridine (4-MPY)- \\ functionalized silver nanoparticles (AgNPs) based on the interaction between protamine and trypsin. \\ The polycationic protamine not only served as a substrate for enzyme hydrolysis but also worked as a \\ medium for SERS enhancement, which could bind negatively charged 4-MPY-functionalized AgNPs and \\ induce their aggregation. The hydrolysis catalyzed with trypsin in sample solution decreased the \\ concentration of free protamine, resulting in the dispersion of AgNPs and thus decreasing the Raman \\ intensity of 4-MPY, by which the trypsin could be sensed optically. A detection level down to $0.1 \mathrm{ng}$ \\ $\mathrm{mL}^{-1}$ for trypsin was obtained. The induced accumulation of AgNPs modified with Raman reporter \\ 4-MPY largely enhanced the SERS responses. A good linearity was found within the wide range over \\ five orders of magnitude and reasonable relative standard deviations (between 2.4 and 11.6\%) were \\ attained. By using trypsin as a model, the new concept can provide an excellent platform for \\ ultrasensitive SERS measurements of various proteases/enzymes which can lead to nanoparticles \\ stability change through catalyzed hydrolysis toward substrate.
}

\section{Introduction}

Proteases, as paradigms of enzyme catalysis, play vital roles in biological and physiological processes including digestion, blood clotting, immune response, and a variety of cellular activities. ${ }^{1-4}$ In addition, as the common agents to many viruses and infectious diseases, they are involved in a host of pathological processes. ${ }^{5-10}$ Therefore, the simple and timely detection of proteases activity is often critical. As a consequence, substantial efforts have been devoted toward the development of various chemo/biosensors to identify and quantify proteases, such as spectrophotometry, ${ }^{11}$ fluorimetry, ${ }^{12}$ chromatography, ${ }^{13}$ electrochemistry ${ }^{\mathbf{1 4}}$ and immunology. ${ }^{\mathbf{1 5}}$ However, most of them are laborious and complicated, especially difficult in designing materials with suitable structure to interact with proteases with detectable signals. Therefore, it is highly desirable to develop simple, rapid, sensitive and selective methods for detection of proteases.

\footnotetext{
${ }^{a}$ Key Laboratory of Coastal Zone Environmental Processes and Ecological Remediation, Yantai Institute of Coastal Zone Research, Chinese Academy of Sciences, Yantai, 264003, P. R. China. E-mail: lxchen@yic.ac.cn; Fax: +86 535-2109130; Tel: +86 535-2109130

${ }^{b}$ University of Chinese Academy of Sciences, Beijing, 100049, P. R. China

$\dagger$ Electronic supplementary information (ESI) available: Additional information as noted in text. See DOI: 10.1039/c3nr00637a
}

Surface-enhanced Raman scattering (SERS) technique has received increasing attention and become a powerful spectroscopy technique owing to its narrow bandwidth, evading photobleaching as well as the ability to perform multiplexed analysis using a single laser wavelength, since its discovery in the late 1970s. ${ }^{\mathbf{1 6 - 1 9}}$ Although traditional Raman spectroscopy is limited by low sensitivity, SERS could provide a significant signal enhancement with an enhancement factor of $10^{6}$ to $10^{14}$ at or near the rough metal surface. ${ }^{20}$ Meanwhile, nanomaterials have become a type of promising SERS substrates since they can effectively enhance Raman signals of adsorbed Raman reporter molecules. ${ }^{\mathbf{1 6}}$ Silver nanoparticles (AgNPs) are recommended as enhanced substrates due to their strong SERS activity, and they can be easily synthesized and modified by different agents. ${ }^{21} \mathrm{Up}$ to now, various AgNPs-based SERS applications have been reported. ${ }^{\mathbf{1 6} 21-24}$ For example, Ren et al. have reported the determination of mercury ions by a direct SERS technique based on the interaction between AgNPs and mercury ions. ${ }^{21}$ Jiang et al. have developed a AgNPs aggregates-based SERS substrate for sensitive detection of polycyclic aromatic hydrocarbons. $^{22}$ Our group has developed a highly sensitive SERS strategy for detection of arsenic ions by using glutathione functionalized AgNPs. ${ }^{23}$ In general, SERS-based assay is conducted by two modes, i.e., via controlling distance between reporter and substrate, and based on aggregation. ${ }^{24}$ However, to 
the best of our knowledge, there have been no examples based on anti-aggregation of AgNPs for SERS detection of proteases.

Here, we expect to develop a simple and effective SERS strategy based on anti-aggregation of 4-mercaptopyridine (4-MPY)-functionalized AgNPs with the aid of polycationic protamine for detection of protease. Protamine, a low molecular weight protein, has $\sim 20$ positive charges as it is rich in basic arginine residues. ${ }^{25}$ The polycation structure of protamine allows the strong electrostatic binding with polyanionic heparin $^{26,27}$ as well as the negatively charged nanoparticles, ${ }^{27}$ such as gold nanoparticles and AgNPs. It should be noted that the interaction between protamine and negatively charged AgNPs could lead to the aggregation of AgNPs and thus induce the changes in colors and surface plasmon resonance absorption. In this case, the AgNPs have strong Raman enhancement effect for Raman reporter. On the other hand, if polycationic protamine is cleaved to smaller fragments, the interaction between fragmentary protamine and AgNPs will be too weak so that it cannot cause the aggregation of AgNPs. Consequently, the Raman enhancement effect of AgNPs would be decreased. Herein, trypsin was adopted as a protease model, since it is the most important digestive enzyme which plays a critical role in controlling pancreatic exocrine function. ${ }^{28}$ Protamine was successfully used as the substrate for trypsin cleaving polypeptide based on the specific proteolysis property of trypsin toward protamine. ${ }^{4}$ The developed SERS method was demonstrated to be highly sensitive toward trypsin, and capable of accurate quantification in a broad linear range. Furthermore, the proposed SERS-based concept might be well applied to monitoring various targets by virtue of target hydrolysis catalyzed-induced nanoparticles stability changes.

\section{Experimental section}

\section{Reagents}

Trypsin from bovine pancreas (13 $000 \mathrm{U} \mathrm{mg}^{-1}$ ), pepsin from porcine gastric mucosa ( $\geq 250$ units per $\mathrm{mg}$ solid), lysozyme from chicken egg white $\left(\sim 100000 \mathrm{U} \mathrm{mg}^{-1}\right)$, glucose oxidase from Aspergillus niger Type X-S (100 000-250 000 units per g), thrombin from bovine plasma (40-300 NIH units per $\mathrm{mg}$ protein), 4-mercaptopyridine (4-MPY) and protamine sulfate salt were purchased from Sigma-Aldrich (USA). Rhodamine 6G (R6G) was obtained from Aladdin Chemistry Co. Ltd (Shanghai, China). Malachite green (MG) was purchased from Tianjin Kermel Chemical Reagent Co. Ltd (Tianjin, China). Silver nitrate $\left(\mathrm{AgNO}_{3}, 99.8 \%\right)$, sodium hydroxide $(\mathrm{NaOH}, 96 \%)$, hydrogen tetrachloroaurate(III) $\left(\mathrm{HAuCl}_{4}\right)$ and trisodium citrate were obtained from Sinopharm Chemical Reagent (Shanghai, China). Hydroxylamine hydrochloride $\left(\mathrm{NH}_{2} \mathrm{OH} \cdot \mathrm{HCl}\right)$ was purchased from Tianjin Kermel Chemical Reagent Co. Ltd. (Tianjin, China). 4-(2-Hydroxyethyl)-1-piperazine ethanesulfonic acid (HEPES) was provided by Shanghai Shengong Co. (Shanghai, China). All other solvents and reagents were of analytical grade and used without further purification. HEPES buffer solution used for all experiments was adjusted with $1 \mathrm{M}$ $\mathrm{NaOH}$ to $\mathrm{pH}$ 7.4. Deionized water with $18.2 \mathrm{M} \Omega$ specific resistances obtained by a Pall Cascada laboratory water system was used throughout.

\section{Instrumentation}

All measurements of SERS spectra were carried out on a Thermo Scientific RFS100 Raman system equipped with a microscope and a $632.8 \mathrm{~nm}$ diode pumped He-Ne laser source with a power of $5 \mathrm{~mW}$. The transmission electron microscopy (TEM) analyses were acquired on a JEM-1230 electron microscope (JEOL, Ltd., Japan) with an accelerating voltage of $100 \mathrm{kV}$. The zeta potential data were collected by using a Zetasizer Nano ZS-90 (Malvern Instruments, Malvern, UK) with 2-fold diluted measuring sample mixtures.

\section{Preparation of nanoparticles}

Hydroxylamine ions capped AgNPs. Hydroxylamine ions capped AgNPs were prepared by the reduction of silver nitrate with hydroxylamine hydrochloride at room temperature following the process described with necessary modification. ${ }^{29}$ Briefly, $10.44 \mathrm{mg} \mathrm{NH} \mathrm{N}_{2} \mathrm{OH} \cdot \mathrm{HCl}$ was dissolved in $89 \mathrm{~mL}_{2} \mathrm{O}$ and was mixed with $1 \mathrm{~mL}$ of $0.30 \mathrm{M} \mathrm{NaOH}$ to maintain an initial alkaline $\mathrm{pH}$. Then, $10 \mathrm{~mL}$ of $0.01 \mathrm{M} \mathrm{AgNO}_{3}$ solution was added to the above solution under stirring. The reaction mixtures were continuously stirred for another one hour. The final prepared silver colloid was stored at room temperature. The UV-vis spectroscopy was used to confirm the typical surface plasmon resonance peak at $412 \mathrm{~nm}$ (Fig. S1 $\dagger$ ). TEM was used to characterize the morphology and particle size of the produced colloids, and the average particle size was estimated to be $35 \pm$ $5 \mathrm{~nm}$ in diameter. The zeta potential of hydroxylamine ions capped AgNPs was determined as $-36.2 \mathrm{mV}$, which confirmed that they were negatively charged.

Citrate capped AgNPs. The citrate capped AgNPs were synthesized based on a previous method. ${ }^{30}$ Typically, $1.06 \mathrm{~mL}$ of $\mathrm{AgNO}_{3}(0.10 \mathrm{M})$ was added to $100 \mathrm{~mL}$ of water at $45^{\circ} \mathrm{C}$ and the solution was heated rapidly to boiling. Then $2 \mathrm{~mL}$ of trisodium citrate $(1 \%)$ was injected under vigorous stirring, and the solution was kept on boiling for $45 \mathrm{~min}$. The resulting solution was cooled at room temperature. TEM image of the obtained citrate capped AgNPs was shown in Fig. S2A. $\dagger$

Gold/silver core-shell nanoparticles (Au@Ag NPs). The silver coated gold nanoparticles (Au@Ag NPs) were prepared by reduction of $\mathrm{Ag}^{+}$in the presence of Au seed approach following the recipe of Natan's group with slight modifications. ${ }^{31,32}$ First, the Au seed with $c a .13 \mathrm{~nm}$ in diameter was prepared by adding $10 \mathrm{~mL}$ of $38.80 \mathrm{mM}$ sodium citrate to $100 \mathrm{~mL}$ of boiling $1.00 \mathrm{mM}$ $\mathrm{HAuCl}_{4}$ under vigorous stirring. After appearance of a deep red color, boiling and stirring were continued for another $15 \mathrm{~min}$ under refluxing and then allowed to cool at room temperature. Second, to prepare the Au@Ag NPs, $10 \mathrm{~mL}$ of Au seed was diluted to $100 \mathrm{~mL}$ solution and was reheated to boil under refluxing followed by addition of $1 \mathrm{~mL}$ of $38.80 \mathrm{mM}$ sodium citrate. Then, $5 \mathrm{~mL}$ of $0.01 \mathrm{M} \mathrm{AgNO}_{3}$ was added and the boiling and stirring were continued for another $1 \mathrm{~h}$. The Au@Ag colloidal solution was cooled to room temperature and stored at 
$4{ }^{\circ} \mathrm{C}$ and protected from light. TEM image of the obtained $\mathrm{Au} @ A g$ NPs was shown in Fig. S2B. $\dagger$

\section{Functionalization of AgNPs with 4-MPY}

Six microliters $(6 \mu \mathrm{L})$ of $250 \mu \mathrm{M} 4$-MPY was added to $6 \mathrm{~mL}$ of asprepared AgNPs with stirring for $2 \mathrm{~h}$ and then the mixture was left standing overnight at room temperature.

\section{SERS analysis of trypsin}

All the standard solutions of protamine, trypsin, pepsin, lysozyme, glucose oxidase and thrombin were respectively prepared by using $10 \mathrm{mM}$ HEPES buffer solution ( $\mathrm{pH}$ 7.4).

The samples for trypsin detection were prepared by adding $10 \mu \mathrm{L}$ of trypsin aqueous solution with various concentrations to the solution of $0.3 \mu \mathrm{g} \mathrm{mL} \mathrm{m}^{-1}$ of protamine $(10 \mu \mathrm{L})$, and the obtained mixtures were incubated at room temperature for 5 min, followed by adding $80 \mu \mathrm{L}$ of 4-MPY-modified AgNPs. Then the samples were measured by Raman spectrometry with an exposure time of $4 \mathrm{~s}$ and a laser power of $5 \mathrm{~mW}$.

\section{Safety consideration}

As aqua regia has strong oxidizing capacity and adverse effects on human health, all of the experiments involving aqua regia should be performed with protective glasses and gloves.

\section{Results and discussion}

\section{Analytical principle}

Scheme 1 shows the possible analytical principle of SERS sensing trypsin based on anti-aggregation of the 4-MPY-functionalized AgNPs. As seen, the freshly synthesized AgNPs were well dispersed (Fig. 1A(a)) and displayed a bright yellow color

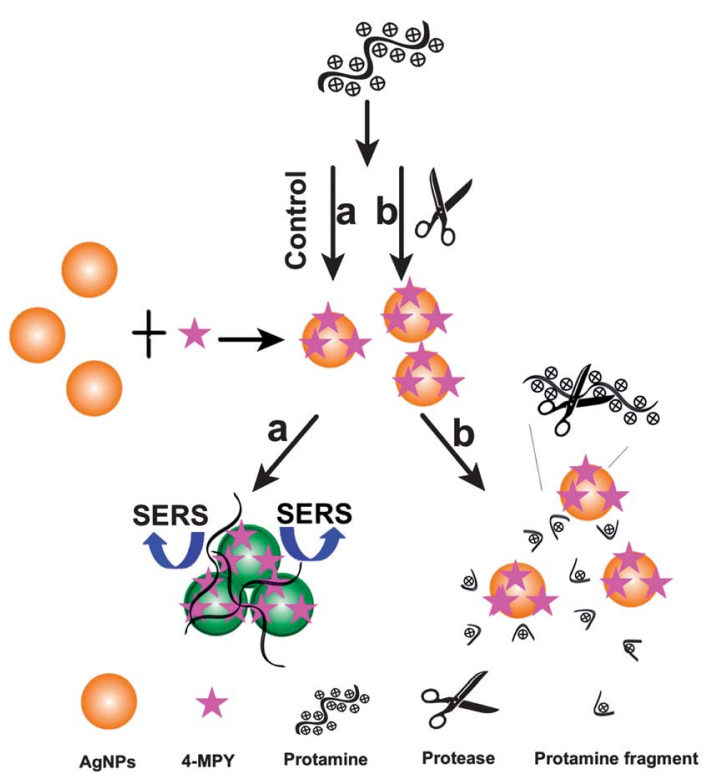

Scheme 1 Schematic representation of the proposed SERS sensing principle for protease detection.

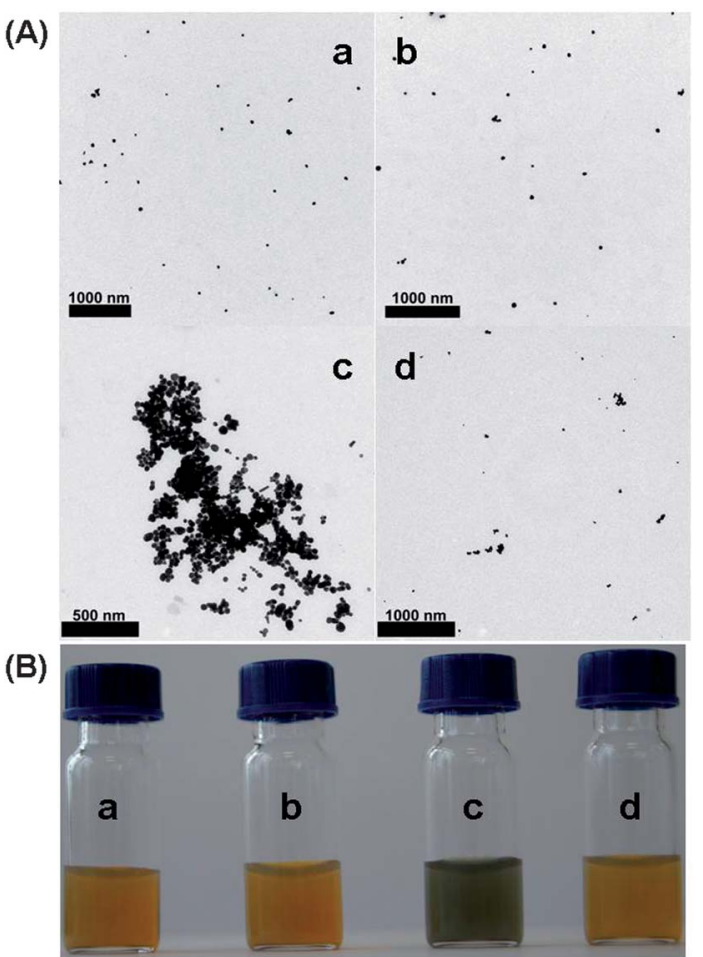

Fig. 1 TEM images (A) and color changes (B) corresponding to (a) AgNPs, (b) AgNPs + 4-MPY, (c) AgNPs + 4-MPY + protamine and (d) AgNPs + 4-MPY + protamine + trypsin

(Fig. 1B(a)). During the process of SERS probe synthesis, the added appropriate amounts of the Raman reporter, 4-MPY, replaced parts of the hydroxylamine ions and were adsorbed on AgNPs, and then the surface of AgNPs kept both 4-MPY and hydroxylamine ions. In this case, strong Raman signals would be produced owing to the presence of 4-MPY, and the existence of hydroxylamine ions would keep the stability of AgNPs in solutions. Generally speaking, 4-MPY interacts with AgNPs through three different ways, namely, via the lone pair electrons of the sulfur $(\mathrm{S})$ or nitrogen $(\mathrm{N})$ atom, or via the aromatic $\pi$ electrons. According to the previous work, ${ }^{33}$ it could be concluded that the $\mathrm{Ag}-\mathrm{S}$ interaction was the major contribution to chemisorption between 4-MPY and AgNPs, the Ag-N interaction played a secondary role in the adsorption process which was variable with the surrounding $\mathrm{pH}$ conditions, while the surface-aromatic $\pi$ interaction was not important. In this work, the Ag-S interaction was characterized by the disappearance of a band near $2575 \mathrm{~cm}^{-1}$ due to the $\mathrm{SH}$ stretching mode as well as the downward shift of $721 \mathrm{~cm}^{-1}(\beta(\mathrm{CC}) / \nu(\mathrm{C}-\mathrm{S}))$ to about $707 \mathrm{~cm}^{-1}$ and the remarkable enhancement of 1096 $\mathrm{cm}^{-1}$ (ring breathing/C-S), ${ }^{33,34}$ as shown in Fig. S3. $\dagger$ The weak bands at $1221 \mathrm{~cm}^{-1}(\beta(\mathrm{CH}) / \delta(\mathrm{NH}))$ and $1579 \mathrm{~cm}^{-1}(\nu(\mathrm{CC}))$ and the emergence of the bands at $1611 \mathrm{~cm}^{-1}(\nu(\mathrm{CC}))$ were the marker bands for $\mathrm{N}$-protonation (Fig. S3†). ${ }^{33,34}$ As is well known that the pyridinium cation could not be adsorbed efficiently when pyridine was adsorbed on the silver surface. ${ }^{33}$ Thus, herein, Ag-S bond was the main interaction between 4-MPY and AgNPs. 
Upon adjusting the added amounts of 4-MPY, the color of colloid AgNPs remained bright yellow (Fig. 1B(b)), indicating that the 4-MPY-modified AgNPs were still monodisperse (Fig. 1A(b)). After addition of protamine, a polypeptide rich in arginine residues with a charge of about $+20,,^{35}$ it would be adsorbed on the surface of 4-MPY-modified AgNPs through electrostatic interaction which would neutralize the negatively charged hydroxylamine ions capped AgNPs. This interaction between protamine and AgNPs reduced the stability of hydroxylamine ions-stabilized AgNPs, and thus resulted in a dramatic aggregation of AgNPs as well as an obvious color change (Fig. 1A(c) and B(c)). Meanwhile, the aggregated AgNPs ingeniously produced a number of "hot spots" owing to huge electromagnetic field enhancements at nanoscale junctions and interstices, ${ }^{32}$ so the Raman signals excited by laser were greatly enhanced. However, excitingly, once trypsin was present, SERS signals would be significantly reduced (Scheme 1) along with a native bright yellow color (Fig. 1B(d)), by which the trypsin could be sensed. The reason might well be attributed to that trypsin as an important and typical protease can catalyze the hydrolysis of protamine, and thereby decreasing the concentration of protamine in the solution and discouraging the aggregation of AgNPs (Fig. 1A(d)), as illustrated in Scheme 1.

To confirm the feasibility of this system for trypsin detection, a series of control experiments were carried out and the results are shown in Fig. 2. It could be seen clearly that in the presence of separate AgNPs, 4-MPY, protamine, or any two of them, there were almost no SERS signals (Fig. 2, curve a-f), while strong SERS spectrum (Fig. 2, curve h) was obtained from the sample with AgNPs, 4-MPY and protamine. But in the presence of trypsin, there was a significant decrease in the SERS intensity (Fig. 2, curve g), suggesting that AgNPs aggregation degree was remarkably decreased owing to the hydrolysis effect of trypsin to protamine. All the results were consistent with that we anticipated and confirmed the feasibility of the SERS assay for detection of trypsin.

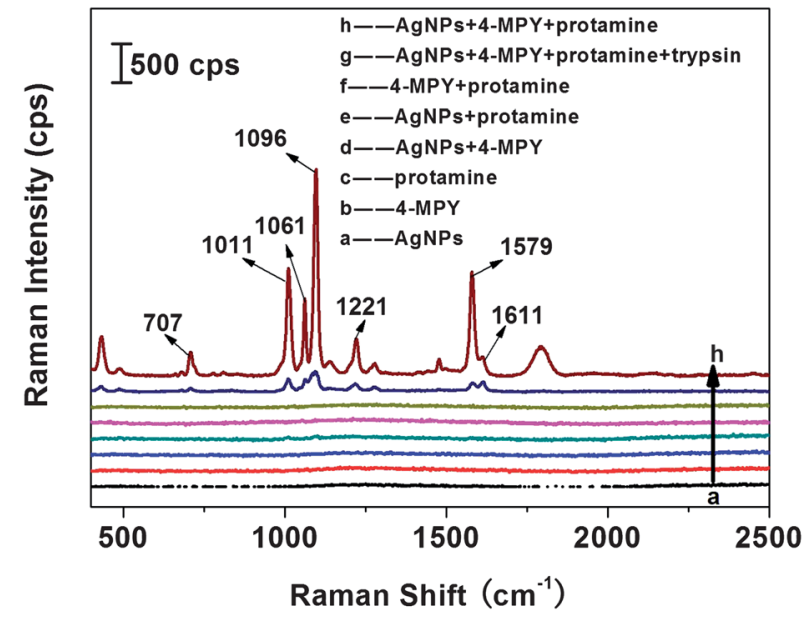

Fig. 2 Raman spectra of (a) AgNPs, (b) 4-MPY, (c) protamine, (d) AgNPs + 4-MPY, (e) AgNPs + protamine, (f) 4-MPY + protamine, (g) AgNPs + 4-MPY + protamine + trypsin and (h) AgNPs + 4-MPY + protamine.

\section{Parameters optimization}

It has been well-established that the SERS substrates have an important effect on the SERS enhancement. ${ }^{\mathbf{1 6}}$ The most frequently used silver colloids are synthesized either by reduction of silver nitrate with sodium citrate so-32 $^{32}$ by reduction of silver nitrate with hydroxylamine hydrochloride. ${ }^{29}$ Citrate reduction of silver nitrate requires adding the reducing agent to the silver nitrate solution along with vigorous stirring and refluxing. ${ }^{29}$ During this synthesis process, due care must be conducted, and otherwise, the colloid would aggregate and eventually precipitate the metal, which would lead to changing properties of the silver colloid and thus also varying enhancement factors. ${ }^{29}$ However, excitingly, the hydroxylamine-reduced silver colloid was prepared at room temperature within a short time with high preparation success rates while maintaining good reproducibility in the obtained enhancement factors and could be used immediately after preparation. ${ }^{29}$ Furthermore, as shown in Fig. S4, $\uparrow$ the highest SERS enhancement could be obtained when hydroxylamine ions capped AgNPs were applied. So, the hydroxylamine ions capped AgNPs were selected throughout the study.

Then, three kinds of Raman reporters were studied including 4-MPY, R6G and MG. As seen from Fig. S5, $\uparrow$ 4-MPY exhibited strong SERS signals but there were almost none for the other two reporters. This might be owing to the fact that the Raman reporters had different types of bindings with AgNPs. As one kind of thio small molecules, 4-MPY was bonded with AgNPs mainly through strong $\mathrm{Ag}-\mathrm{S}$ interaction in this system. ${ }^{16,33,34}$ While, R6G and MG were cationic dyes and they were bonded with AgNPs primarily by weak electronic interactions. ${ }^{16,36,37}$ We further investigated the zeta potentials of AgNPs, AgNPs + 4-MPY, AgNPs + R6G and AgNPs + MG, which were $-36.2 \mathrm{mV},-35.5 \mathrm{mV},-29.5 \mathrm{mV}$ and $-29.8 \mathrm{mV}$, respectively. The data suggested that R6G and MG could greatly reduce the surface negative charges of AgNPs thereby weaken the interaction between AgNPs and polycation protamine and decrease the SERS responses. It should be noted that protamine could also powerfully compete with R6G and MG, since their respective binding with AgNPs were all through electronic interactions. In this system, except protamine, neither R6G nor MG could cause the aggregation of AgNPs. So, it is very likely that the polycation protamine would easily replace the Raman reporters of R6G and MG adsorbed on AgNPs, and would remarkably reduce the Raman enhancing signals. Meanwhile, it was found that 4-MPY had little effect on the negative charges of AgNPs, and thereby without affecting the binding between protamine and AgNPs. Since the Ag-S interaction is much stronger than the electronic interaction, 4-MPY would not be replaced by protamine. Therefore, in the presence of protamine, the 4-MPY modified on AgNPs provided strong Raman enhancing signals. In this study, 4-MPY was adopted as the Raman reporter.

Next, the influence of the concentration of 4-MPY on the SERS intensity was investigated with four different concentrations (Fig. S6A $\dagger$ ). It was found that the higher the concentration of 4-MPY in the range of $1-2.5 \mu \mathrm{M}$ was, the higher the SERS signal intensity was obtained. However, further increasing the 
concentration of 4-MPY would induce AgNPs aggregation and a relatively low Raman signal. Therefore, the concentration of $2.5 \mu \mathrm{M}$ was chosen.

Finally, we studied the effect of the amount of protamine for detection of trypsin. As seen from Fig. S6B, $\dagger$ the SERS signal became large with increasing the concentration of protamine because of the enhanced aggregation of AgNPs, while at concentration $>0.3 \mu \mathrm{g} \mathrm{mL} \mathrm{m}^{-1}$, the SERS signal presented a significant decrease. Further increasing the concentration to $5 \mu \mathrm{g} \mathrm{mL}{ }^{-1}$, the SERS signal was almost the same as that in the absence of protamine, and the color of the AgNPs colloid also recovered to native bright yellow, as shown in Fig. S7. $\dagger$ Moreover, the present zeta potential was attained as $+43.5 \mathrm{mV}$. Thus, it could be concluded that the protamine played a role of stabilizer for AgNPs at high concentrations and thereby the developed SERS strategy would be invalid. When the concentration of protamine reached $0.3 \mu \mathrm{g} \mathrm{mL} \mathrm{L}^{-1}$, the Raman intensity was high enough for detection of trypsin (Fig. S6B $\dagger$ ). Therefore, $0.3 \mu \mathrm{g} \mathrm{mL}{ }^{-1}$ was chosen as the optimal concentration of protamine.

\section{Analytical sensitivity and selectivity}

The ability of the developed SERS biosensor for quantitative analysis of trypsin was further evaluated. Under the above optimized parameters, the SERS spectra of 4-MPY on AgNPs to different concentrations of trypsin were recorded for sensitivity investigation. As shown in Fig. 3A, there are many spectral features which are attributed to 4-MPY bands, such as those located at 707, 1011, 1061, 1096, 1221, 1579 and $1611 \mathrm{~cm}^{-1}$. The strong SERS band at $1096 \mathrm{~cm}^{-1}$ corresponding to the ringbreathing/C-S stretching mode indicated that 4-MPY was adsorbed on the surface of AgNPs colloid through the sulfur atom. ${ }^{33,38}$ It is also supported by the stretching mode at $707 \mathrm{~cm}^{-1}$, which displayed an increase in intensity. ${ }^{38,39}$ The bands at 1579 and $1611 \mathrm{~cm}^{-1}$ were assigned to C-C stretching mode, and the bands at 1061 and $1221 \mathrm{~cm}^{-1}$ corresponding to $\mathrm{C}-\mathrm{H}$ mode were also observed. ${ }^{39,40}$ The band at $1011 \mathrm{~cm}^{-1}$ was assigned to ring-breathing mode. ${ }^{41}$ It can be clearly seen that the peak around $1096 \mathrm{~cm}^{-1}$ was the most prominent one, and its Raman intensity had a concomitant significant decrease with the increase of trypsin concentration. Naturally, the process of trypsin-induced anti-aggregation of 4-MPY-functionalized AgNPs was followed by monitoring the changes in Raman intensity value at $1096 \mathrm{~cm}^{-1}$. Thus, the Raman intensity of 4-MPY on AgNPs at $1096 \mathrm{~cm}^{-1}$ was used here for quantitative analysis of trypsin. As shown in Fig. 3B, the Raman intensity exhibited a good linear fit to the logarithm of the trypsin concentration in the range of $0.1-10000 \mathrm{ng} \mathrm{mL}^{-1}$, with relative standard deviations between 2.4 and $11.6 \%$. The minimum detectable concentration was $0.1 \mathrm{ng} \mathrm{mL}^{-1}$, indicating that the SERS strategy was ultrasensitive and highly reliable for the detection of trypsin.

The detection selectivity of the anti-aggregation-based SERS strategy toward trypsin was also investigated relative to other potentially competitive enzymes, including pepsin, thrombin, lysozyme and glucose oxidase. As shown in Fig. 4, in the
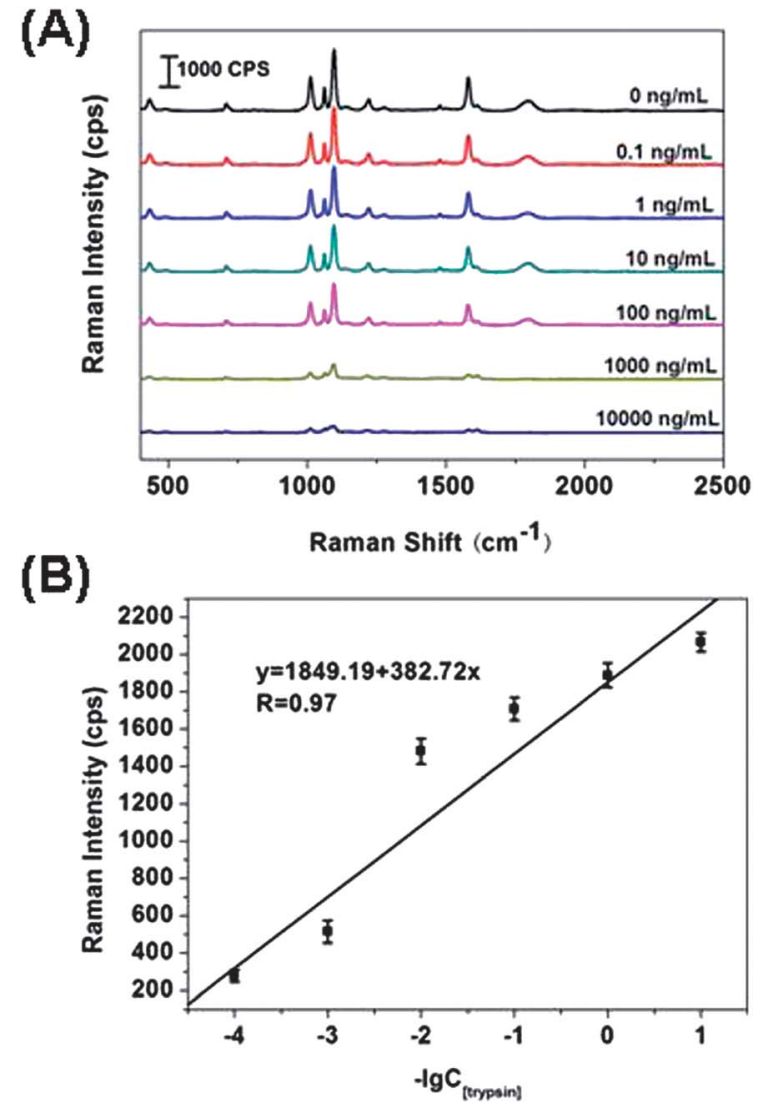

Fig. 3 (A) Raman intensity changes of 4-MPY modified on AgNPs-protamine mixed solution upon addition of different concentrations of trypsin (0-10 $000 \mathrm{ng}$ $\mathrm{mL}^{-1}$ ) and (B) a plot of corresponding intensity of the Raman band at $1096 \mathrm{~cm}^{-1}$ versus the logarithm of trypsin concentration in the range of 0.1 to $10000 \mathrm{ng}$ $\mathrm{mL}^{-1}$. Each error bar represented the standard deviations based on three independent measurements.

presence of pepsin, SERS intensity barely changed compared to that given by the blank, while for thrombin, SERS intensity slightly increased, possibly because it caused the increase of

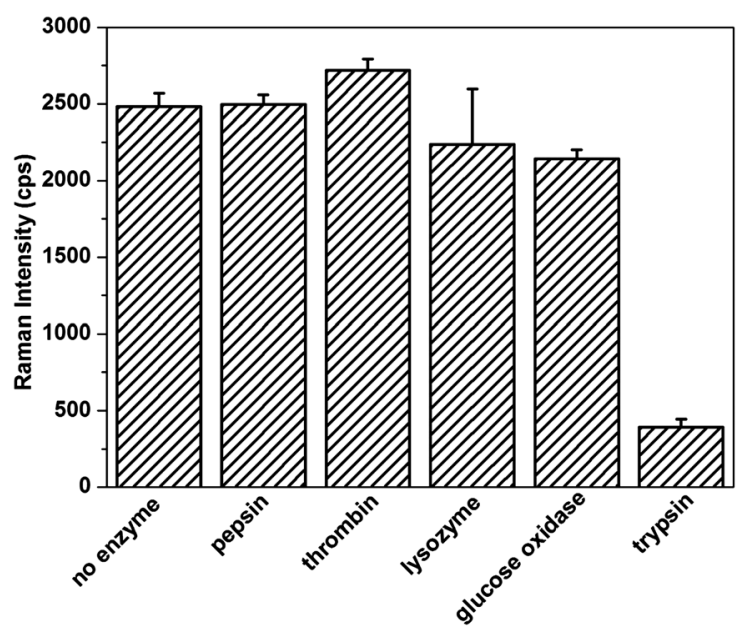

Fig. 4 Comparison of the Raman intensity of 4-MPY modified on AgNPs in the absence or presence of different enzymes. The concentration of each enzyme was $1 \mu \mathrm{gL}^{-1}$. 
AgNPs aggregation degree. Meanwhile, as seen from the figure, after addition of lysozyme and glucose oxidase, the SERS intensity had a slight decrease; interestingly, trypsin led to a significant SERS decrease at an identical concentration with lysozyme and glucose oxidase. This case suggested that only trypsin possessed strong ability to hydrolyze protamine, and thereby hindering AgNPs aggregation as well as facilitating the dispersion of aggregated ones. It demonstrated that the developed program provided attractive specificity toward trypsin.

\section{Analytical performance comparison}

The analytical performance of this developed SERS strategy toward trypsin was compared with some reported colorimetric, fluorescent, and electrochemical methods for trypsin detection. As can be seen from Table $\mathrm{S} 1, \dagger$ the colorimetric methods based on Arg6 inducing the aggregation of gold nanoparticles ${ }^{28}$ or using a derivative of Rhodamine $\mathrm{B}$, copper ions and bovine serum albumin ${ }^{\mathbf{1 1}}$ have the advantages of label-free, visualization or simple operation processes, yet they show lower sensitivities or narrower linear ranges. The fluorimetry methods possess relatively wider quantitation spans, but they are also involved in the problems of complicated synthesis and purification procedures or lower sensitivities. ${ }^{\mathbf{4 1 - 4 3}}$ The electrochemistry methods have the problems of time-consuming processes in production of electrodes and lower sensitivities. ${ }^{7,14}$ Our newly developed SERS method based on anti-aggregation of 4-MPY-functionalized AgNPs presents lots of attractive analytical features such as simple operation process, wide linear range, high sensitivity and short analysis time. This SERS strategy is easily performed and can be used for rapid analysis of trypsin at $\mathrm{ng} \mathrm{mL}^{-1}$ level.

\section{Conclusions}

In conclusion, a simple, rapid, sensitive, selective and reproducible SERS assay strategy for determination of trypsin was developed based on anti-aggregation of 4-MPY-functionalized AgNPs. By taking advantages of the significant SERS enhancement properties of AgNPs to Raman reporter 4-MPY and the specific proteolysis features of trypsin toward protamine, excellent analytical performances such as high sensitivity (0.1 $\left.\mathrm{ng} \mathrm{mL} \mathrm{mL}^{-1}\right)$ and wide linear range $\left(0.1-10000 \mathrm{ng} \mathrm{mL}^{-1}\right)$ were attained. Since many nanomaterials and Raman reporter molecules as well as specific interactions between targets and substrates have been exploited and some of them are even commercially available, such a sensing strategy can provide an excellent optical sensing platform for SERS analysis of proteases and hold promising potentials for fabrication of a number of chemo/biosensors for various proteases/enzymes based on target-induced nanoparticles stability changes.

\section{Acknowledgements}

This work was financially supported by the National Natural Science Foundation of China (21275158), the Innovation Projects of the Chinese Academy of Sciences (KZCX2-EW-206), and the 100 Talents Program of the Chinese Academy of Sciences.

\section{Notes and references}

1 E. W. Davie, K. Fujikawa and W. Kisiel, Biochemistry, 1991, 30, 10363-10370.

2 F. Cleemann and P. Karuso, Anal. Chem., 2008, 80, 4170-4174.

3 S. S. Shekhawat, J. R. Porter, A. Sriprasad and I. Ghosh, J. Am. Chem. Soc., 2009, 131, 15284-15290.

4 L. Hedstrom, Chem. Rev., 2002, 102, 4501-4523.

5 E. Liaudet-Coopman, M. Beaujouin, D. Derocq, M. Garcia, M. Glondu-Lassis, V. Laurent-Matha, C. Prebois, H. Rochefortand and F. Vignon, Cancer Lett., 2006, 237, 167-179.

6 W. G. Stetler-Stevenson and A. E. Yu, Semin. Cancer Biol., 2001, 11, 143-152.

7 K. L. Gemene and M. E. Meyerhoff, Anal. Biochem., 2011, 416, 67-73.

8 M. Hidalgo and S. G. Eckhardt, J. Natl. Cancer Inst., 2001, 93, 178-193.

9 R. B. Singh, S. P. Dandekar, V. Elimban, S. K. Gupta and N. S. Dhalla, Mol. Cell. Biochem., 2004, 263, 241-256.

10 F. Molinari, V. Meskanaite, A. Munnich, P. Sonderegger and L. Colleaux, Hum. Mol. Genet., 2003, 12, R195-R200.

11 X. D. Lou, L. Y. Zhang, J. G. Qin and Z. Li, Langmuir, 2010, 26, 1566-1569.

12 Y. Y. Wang, Y. Zhang and B. Liu, Anal. Chem., 2010, 82, 86048610.

13 I. Miksik, K. Lacinova, Z. Zmatlikova, P. Sedlakova, V. Kral, D. Sykora, P. Rezanka and V. Kasicka, J. Sep. Sci., 2012, 35, 994-1002.

14 Y. Chen, J. W. Ding and W. Qin, Bioelectrochemistry, 2012, 88, 144-147.

15 I. S. Blair and D. A. McDowell, Curr. Microbiol., 1995, 31, 180185.

16 Y. Q. Wang, B. Yan and L. X. Chen, Chem. Rev., 2013, 113, 1391-1428.

17 L. Wu, Z. Wang, S. Zong, Z. Huang, P. Zhang and Y. Cui, Biosens. Bioelectron., 2012, 38, 94-99.

18 L. X. Chen and J. B. Choo, Electrophoresis, 2008, 29, 18151828.

19 Y. Q. Wang, L. X. Chen and P. Liu, Chem.-Eur. J., 2012, 18, 5935-5943.

20 W. Ren, Y. Fang and E. Wang, ACS Nano, 2011, 5, 6425-6433. 21 W. Ren, C. Zhu and E. Wang, Nanoscale, 2012, 4, 5902-5909. 22 X. Jiang, Y. Lai, M. Yang, H. Yang, W. Jiang and J. Zhan, Analyst, 2012, 137, 3995-4000.

23 J. L. Li, L. X. Chen, T. T. Lou and Y. Q. Wang, ACS Appl. Mater. Interfaces, 2011, 3, 3936-3941.

24 G. Q. Wang, Y. Q. Wang, L. X. Chen and J. B. Choo, Biosens. Bioelectron., 2010, 25, 1859-1868.

25 B. K. Jena and C. R. Raj, Biosens. Bioelectron., 2008, 23, 12851290.

26 X. L. Fu, L. X. Chen, J. H. Li, M. Lin, H. Y. You and W. H. Wang, Biosens. Bioelectron., 2012, 34, 227-231.

27 X. L. Fu, L. X. Chen and J. H. Li, Analyst, 2012, 137, 36533658.

28 W. Xue, G. Zhang and D. Zhang, Analyst, 2011, 136, 31363141. 
29 N. Leopold and B. Lendl, J. Phys. Chem. B, 2003, 107, 57235727.

30 P. C. Lee and D. Meisel, J. Phys. Chem., 1982, 86, 3391-3395.

31 R. G. Freeman, M. B. Hommer, K. C. Grabar, M. A. Jackson and M. J. Natan, J. Phys. Chem., 1996, 100, 718-724.

32 J. W. Chen, X. P. Liu, K. J. Feng, Y. Liang, J. H. Jiang, G. L. Shen and R. Q. Yu, Biosens. Bioelectron., 2008, 24, 66-71.

33 H. S. Jung, K. Kwan and M. S. Kim, J. Mol. Struct., 1997, 407, 139-147.

34 J. W. Hu, B. Zhao, W. Q. Xu, B. F. Li and Y. G. Fan, Spectrochim. Acta, Part A, 2002, 58, 2827-2834.

35 A. Shvarev and E. Bakker, J. Am. Chem. Soc., 2003, 125, 11192-11193.

36 M. Futamata, Y. Y. Yu, T. Yanatori and T. Kokubun, J. Phys. Chem. C, 2010, 114, 7502-7508.
37 U. Nickel, P. Halbig, H. Gliemann and S. Schneider, Ber. Bunsen-Ges. Phys. Chem., 1997, 101, 41-49.

38 Y. F. Wang, Z. H. Sun, H. L. Hu, S. Y. Jing, B. Zhao, W. Q. Xu, C. Zhao and J. R. Lombardi, J. Raman Spectrosc., 2007, 38, 3438.

39 H. Guo, L. Ding and Y. J. Mo, J. Mol. Struct., 2011, 991, 103107.

40 W. Q. Wang, W. L. Li and R. F. Zhang, Mater. Chem. Phys., 2010, 124, 385-388.

41 K. H. Xu, F. Liu, J. Ma and B. Tang, Analyst, 2011, 136, 11991203.

42 H. L. Fan, X. H. Jiang, T. Zhang and Q. H. Jin, Biosens. Bioelectron., 2012, 34, 221-226.

43 L. Z. Hu, S. Han, S. Parveen, Y. L. Yuan, L. Zhang and G. B. Xu, Biosens. Bioelectron., 2012, 32, 297-299. 